\title{
Graded-Index Plastic Optical Fiber with High Mechanical Properties Enabling Easy Network Installations. I
}

\author{
Takaaki Ishigure, ${ }^{1}$ Miki Hirai, ${ }^{2}$ Masataka Sato, ${ }^{2}$ Yasuhiro Koike ${ }^{1}$ \\ ${ }^{1}$ Faculty of Science and Technology, Keio University, 3-14-1, Hiyoshi, Kohoku-ku, Yokohama 223-8522, Japan \\ ${ }^{2}$ Japan Science and Technology Corporation, ERATO, 144-8, Ogura, Saiwai-ku, Kawasaki 212-0054, Japan
}

Received 25 November 2002; accepted 10 April 2003

\begin{abstract}
A doped poly(methyl methacrylate) (PMMA)based graded-index plastic optical fiber (GI POF) with high mechanical strength is reported for the first time. Although the POF is generally believed to have a good mechanical flexibility even if it has a large-core diameter, such a high mechanical strength has been provided by making the polymer chains in the POF highly oriented in its axial direction. If such an orientation of polymer chains is eliminated, the POF becomes brittle, which is similar to silica-based fibers. On the other hand, too high an orientation of the polymer chains induces fiber deformation in a high-temperature at-
\end{abstract}

mosphere resulting from orientation relaxation. This study reports how high mechanical strengths such as the tensile strength and the large elongation are provided to the GI POF. By selecting the optimum heat-drawing conditions, the GI POF has a mechanical strength comparable to that of the commercially available step index (SI) POF. (C) 2003 Wiley Periodicals, Inc. J Appl Polym Sci 91: 404-409, 2004

Key words: mechanical properties; additives; plastic optic fibers; refractive index; poly(methyl methacrylate) (PMMA)

\section{INTRODUCTION}

Single-mode silica fibers are widely utilized in longhaul telecommunications networks, and their use is expanding to include the metropolitan area networks, whereas many short-distance data links favor the lessexpensive multimode fiber technology. Even in the case of silica-based multimode fiber, the core diameter is 50 or $62.5 \mu \mathrm{m}$, which provides greater tolerance in fiber connections than single-mode fibers. Even so, the multimode fiber requires high precision in the alignment at the fiber connection because of the modal noise problem. ${ }^{1}$ Therefore, much interest has been focused on large-core plastic optical fibers (POF) for short-distance data communications area where many fiber connections would be required.

We have proposed a high-bandwidth graded-index (GI) POF as one of the promising candidates for a high-speed data transmission medium. ${ }^{2,3}$ The conventional POF has a large diameter with good flexibility, enabling a smaller bending radius than silica-based optical fibers. This is one of the reasons that a POF network is expected to be installed even in home and office networks.

The GI POF prepared by the interfacial-gel polymerization process we have proposed so $\mathrm{far}^{1,2}$ has a quadratic refractive index profile that is formed by the

Correspondence to: T. Ishigure (ishigure@appi.keio.ac.jp).

Journal of Applied Polymer Science, Vol. 91, 404-409 (2004) (C) 2003 Wiley Periodicals, Inc. concentration distribution of a dopant material. Because the dopant is a kind of plasticizer, there is a concern that the GI POF obtained would have poor mechanical strength compared to the commercially available step-index (SI) POF that is mainly composed of poly(methyl methacrylate) (PMMA) homopolymer. Therefore, in this study the effect of dopants on the mechanical strength of the GI POF is investigated in detail for the first time. It was found that even if $20 \mathrm{wt}$ $\%$ of dopant was doped to the PMMA that is the polymer matrix of the GI POF, a tensile strength as high as that of the commercially available SI POF could be obtained if the fiber was drawn with high drawing tension. Because the GI POF we have proposed is obtained by the heat drawing of the GI preform, the heat-drawing tension is a very important parameter for providing good mechanical properties. Therefore, the optimum preparation process was investigated in this study to obtain a GI POF with both high mechanical strength and no length shrinkage.

\section{EXPERIMENTAL}

\section{Fabrication of GI POF}

The GI POF was obtained by the heat drawing of the GI preform whose diameter was 18 to $30 \mathrm{~mm}$. The preform rod, in which the refractive index gradually decreases from the center axis to the periphery, was prepared by the interfacial-gel polymerization technique whose process is described as follows ${ }^{2,4}$ : a PMMA tube, whose outer diameter was 18 to $30 \mathrm{~mm}$ and the inner diameter 


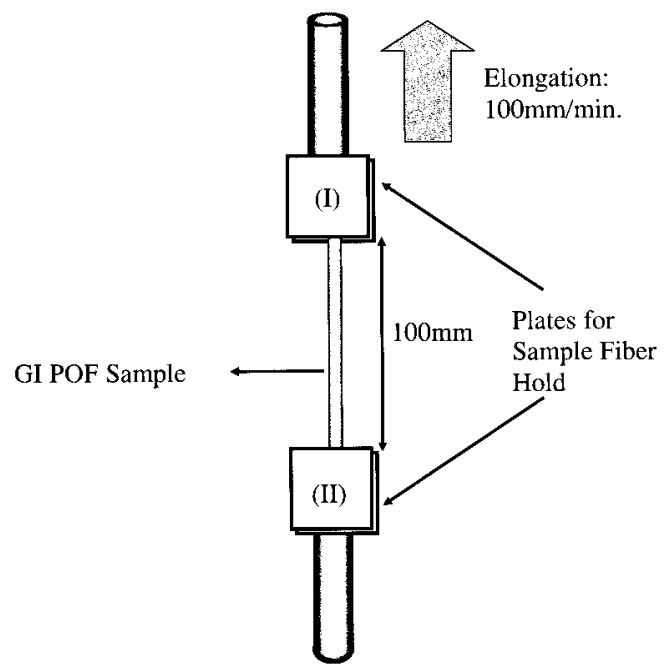

Figure 1 Structure of the GI POF holding part in the mechanical property measurement apparatus.

was $60 \%$ of the outer diameter, was prepared by bulk polymerization from the purified MMA monomer. The PMMA tube was filled with a mixture of MMA monomer, dopant, polymerization initiator, and chain transfer agent. The PMMA tube filled with this monomer mixture was heated from the surrounding environment to induce polymerization. The inner wall of the PMMA tube becomes slightly swollen by the monomer dopant mixture to form the polymer gel phase.

The reaction rate of the polymerization is generally faster in the gel phase because of the "gel effect." Therefore, the polymer phase grows from the inner wall of the tube to the center. During this process, the MMA monomer can diffuse into the gel phase more easily than the dopant molecules because the molecular volume of dopant, which contains benzene rings, is larger than that of monomer. Thus, the dopant molecules are concentrated in the center region of the core to form an almost quadratic refractive index profile. ${ }^{2}$ The GI POF was obtained by the heat drawing of the GI preform. In this study the fiber diameter was set to $600 \mu \mathrm{m}$. Furthermore, the effect of the heat-drawing tension on the mechanical strength was investigated in detail. The GI POFs drawn at the various drawing tensions were obtained from one preform. To vary the drawing tension, the heat-drawing temperature was varied from 180 to $250^{\circ} \mathrm{C}$ during the heat-drawing process. The heat-drawing tension was measured directly by a tension meter during the heat-drawing process. The drawing tension measurement was carried out midway between the bottom of heat-drawing furnace and the fiber takeup reel.

\section{Evaluation of mechanical strength}

The mechanical strength of the GI POF was investigated based on the measurement method of the tensile strength for the commercially available SI POF. ${ }^{5}$ The tensile strengths of the fiber were evaluated by continuously measuring the force that developed as the sample is elongated at a constant rate of extension. The condition is described below in detail. This method was standardized in Japan as JIS C6861-1999. The measurement apparatus is schematically shown in Figure 1. As shown in Figure 1, both ends of a fiber sample were held between two $3 \times 3$-cm plates, and the sample length was approximately $120-130 \mathrm{~mm}$ because the distance from the top of the lower plate (II) to the bottom of the upper plate (I) was set to 100 $\mathrm{mm}$. The sample elongation speed was set to 100 $\mathrm{mm} / \mathrm{min}$. Figure 2 shows a typical stress-strain curve obtained by this tensile strength measurement. From this chart, not only the tensile strength but also the yield stress, elongation at break, and elongation at yield were analyzed.

\section{RESULT AND DISCUSSION}

\section{Tensile strength}

Because $10-20$ wt \% of an aromatic compound is doped in the core region of the GI POF, there is a concern that the tensile strength is influenced not only by the heat-drawing tension but also by the type and concentration of dopant, given that some dopants act as plasticizers. To clarify the effect of dopant on the mechanical strength of the GI POF, the tensile strength of the PMMA homopolymer POF was evaluated first. The PMMA homopolymer POF was also prepared by the heat drawing of a PMMA homopolymer preform

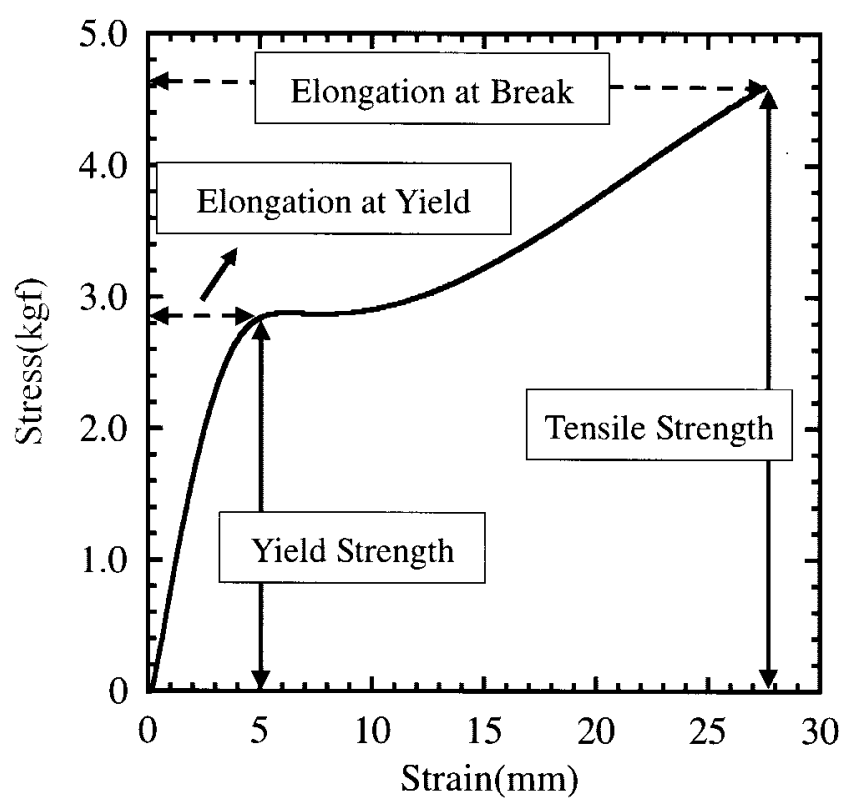

Figure 2 Typical stress-strain curve of the PMMA-based optical fiber and the definitions of several parameters to evaluate the mechanical properties of the GI POF. 


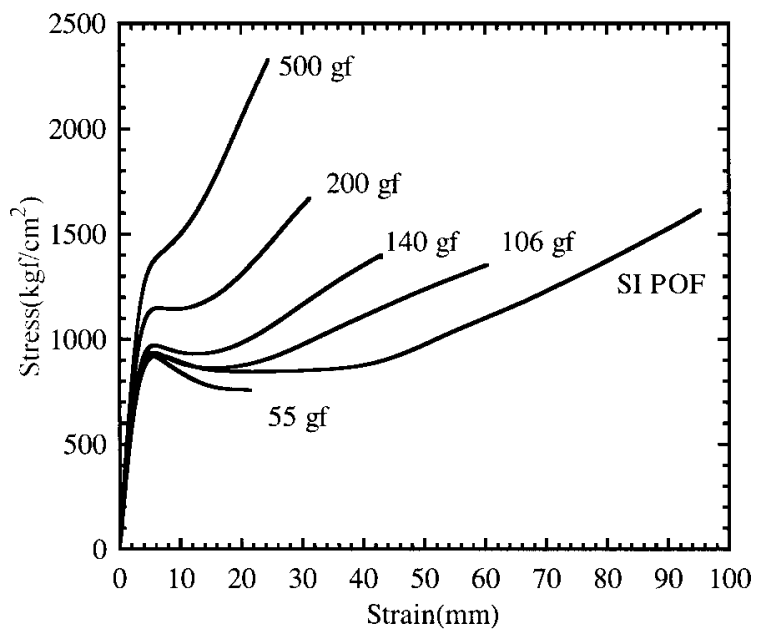

Figure 3 Stress-strain curves of the PMMA homopolymer fibers drawn from the PMMA homopolymer preformed at various drawing tensions.

that was obtained by polymerizing the core region of the PMMA tube with no dopant in the interfacial-gel polymerization process mentioned in the previous section. Five different GI POFs drawn at five different drawing tensions (from 55 to $500 \mathrm{gf}$ ) were prepared for the measurement. The fiber diameter was set to 600 $\mu \mathrm{m}$. The heat-drawing tension is strongly dependent on the structure of the heat-drawing apparatus. Therefore, we tried to draw the fiber by two different types of heat-drawing apparatus at the same drawing tension, and compared their mechanical strength. As a result, there was little difference in the tensile strength. Therefore, the value of the heat-drawing tension is a fixed parameter to discuss how a GI POF with high mechanical strength is produced.

The experimentally measured stress-strain curves are shown in Figure 3. The tensile strength of the PMMA homopolymer POF increases as the drawing tension increases. Because the polymer chains in the POF are more oriented in the axial direction with increasing the heat-drawing tension, a higher tensile strength is observed in the POFs drawn at higher drawing tension. As a comparison, the stress-strain curve of a commercially available SI POF is also shown in Figure 3. The tensile strength of the SI POF was measured as $1600 \mathrm{kgf} / \mathrm{cm}^{2}$, which corresponds to the tensile strength of the PMMA homopolymer POF drawn at $200 \mathrm{gf}$ of drawing tension. It is found from Figure 3 that a PMMA homopolymer POF with higher tensile strength than that of the SI POF is obtained when the fiber was drawn at a tension higher than 200 gf. Other parameters such as the yield stress, elongation at break, and elongation at yield are also strongly dependent on the heat-drawing tension. A detailed analysis is carried out in the next section.

\section{Dopant effect on mechanical strength}

To investigate the effect of the dopant on the mechanical strength of the GI POF in detail, two dopant concentrations (11 and $20 \mathrm{wt} \%$ ) were adopted in this study. The relation between the tensile strength and the heat-drawing tension of the GI POFs doped with 11 and $20 \mathrm{wt} \%$ dopant are shown in Figure 4(a) and (b), respectively. In both Figure 4(a) and (b), the same relation in the PMMA homopolymer fiber is also indicated. Dopants used in these GI POFs were diphenyl sulfide (DPS), diphenyl sulfoxide (DPSO), tricresyl phosphate (TCP), benzyl benzoate (BEN), and triphenyl phosphate (TPP). Even if 20 wt \% dopant was doped in the GI POF, the tensile strength of the GI POF increases with increasing drawing tension, which is the same trend as that of PMMA homopolymer POF. However, if the tensile strengths of the PMMA homopolymer fiber and doped GI POF drawn at the same drawing tension were compared, a lower tensile strength is observed in the doped GI POF, particularly in the $20 \mathrm{wt} \%$-doped one. The higher dopant concentration leads the GI POF to have low tensile strength. Regarding the minimum tensile strength of the $\mathrm{POF}$, the JIS standard specified for the SI POF is 970 $\mathrm{kg} / \mathrm{cm}^{2}$. From Figure $4(\mathrm{~b})$, a GI POF showing a tensile strength higher than $970 \mathrm{~kg} / \mathrm{cm}^{2}$ is prepared by drawing the POF at a tension greater than $100 \mathrm{gf}$, even when the dopant concentration is $20 \mathrm{wt} \%$. It is possible to obtain a GI POF with a sufficient tensile strength to satisfy the JIS specification.

From the results shown in Figure 4(a) and (b), it was found that a high drawing tension increased the tensile strength of the GI POF. However, the high drawing tension of the GI POF caused another problem: a fiber-length shrinkage at high temperature. Because the polymer chains that compose the GI POF are more oriented in its axial direction when it was drawn at a high drawing tension, the GI POF showed a length shrinkage when it was placed in a high-temperature atmosphere because of the orientation relaxation. Figure 5(a) and (b) show the results of the length shrinkage measurement of the GI POF during aging at $70^{\circ} \mathrm{C}$. These results were obtained by measuring the length shrinkage of 1-m GI POFs that were drawn at various tensions, after $48 \mathrm{~h}$ of aging at $70^{\circ} \mathrm{C}$. It is noted that even in the PMMA homopolymer fiber, a length shrinkage was observed during aging if it was drawn at high drawing tension, although the PMMA homopolymer has a glass-transition temperature $\left(T_{g}\right)$ higher than the aging temperature. The GI POF drawn at a high drawing tension also showed a large length shrinkage. Furthermore, the dopant lowers the $T_{g}$ of the core in the GI POF compared to that of the PMMA homopolymer. The relation between the length shrinkage of the GI POF after $48 \mathrm{~h}$ of aging at $70^{\circ} \mathrm{C}$ and the core $T_{g}$ is plotted in Figure 6. In this case, all GI POFs were drawn at a 200-gf drawing tension. The 


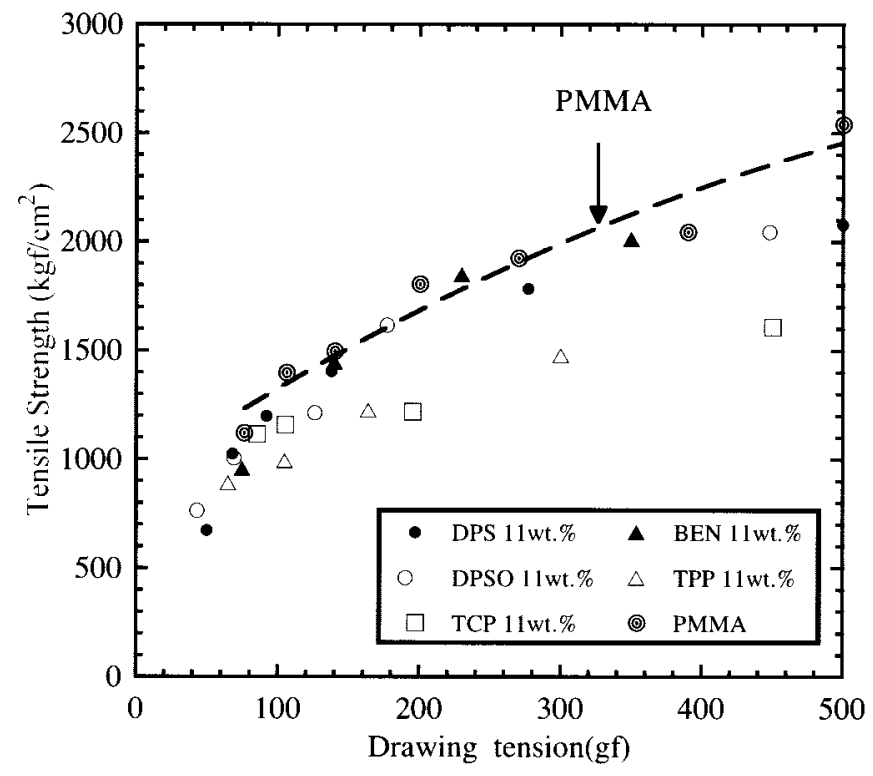

(a)

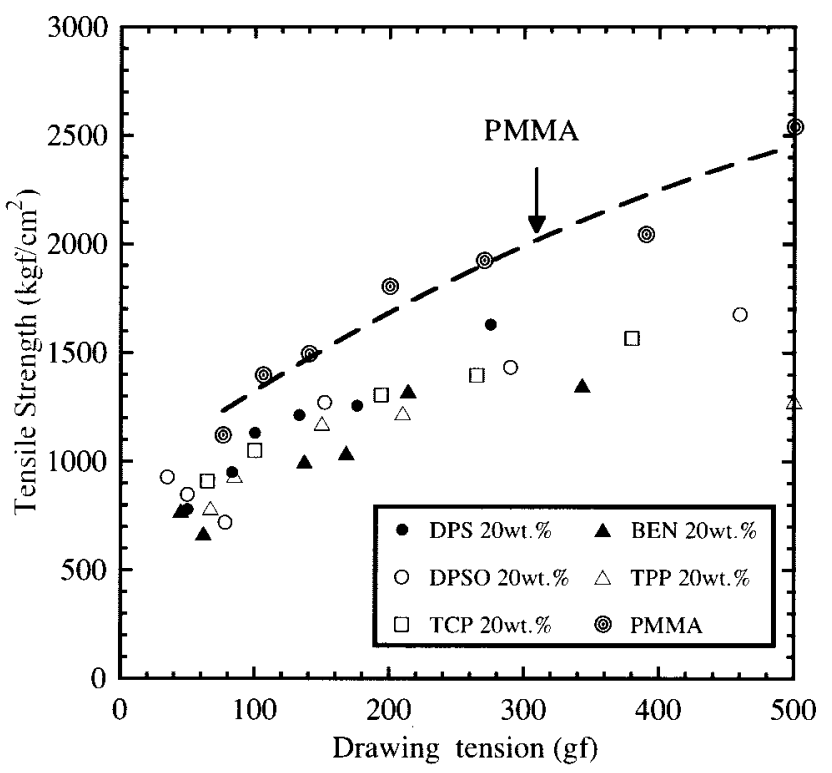

(b)

Figure 4 Relation between the heat-drawing tension and the tensile strength of the GI POF. (a) Dopant concentration in the GI POF is 11 wt \%; (b) Dopant concentration in the GI POF is $20 \mathrm{wt} \%$.

dopant identity and concentration of the GI POFs used in Figure 6 varied widely; thus the core $T_{g}$ exhibits various values. Figure 6 shows an almost linear relation between the length shrinkage of the GI POF and the core $T_{g^{\prime}}$ which is independent of the dopant types. From the results shown in Figures 3-6, it is found that the GI POF with high tensile strength and small length shrinkage (related to the high temperature resistance) is obtained by maintaining a high $T_{g}$ of the core, which is enabled by decreasing the dopant concentration, ${ }^{6}$ and by drawing

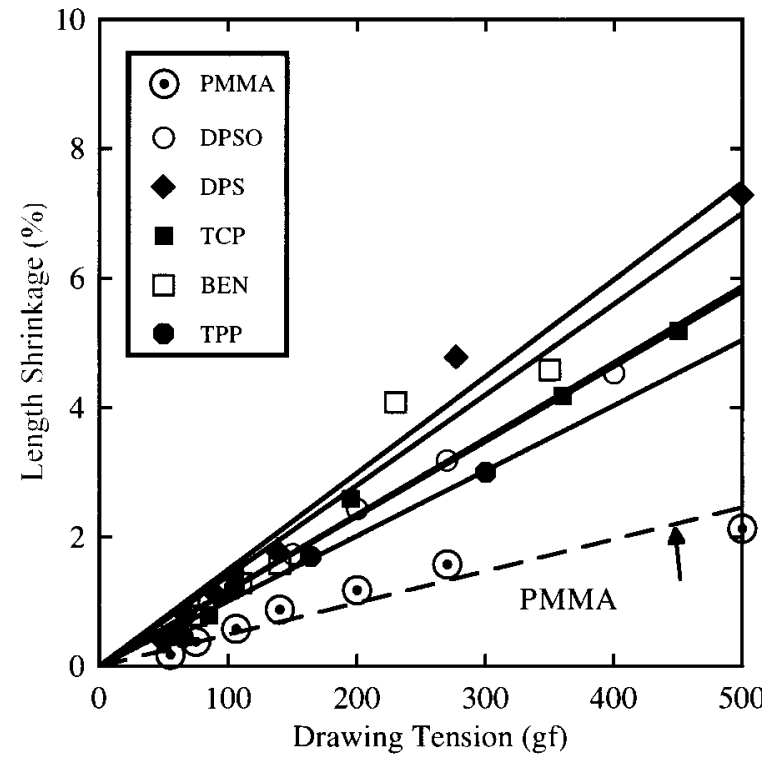

(a)

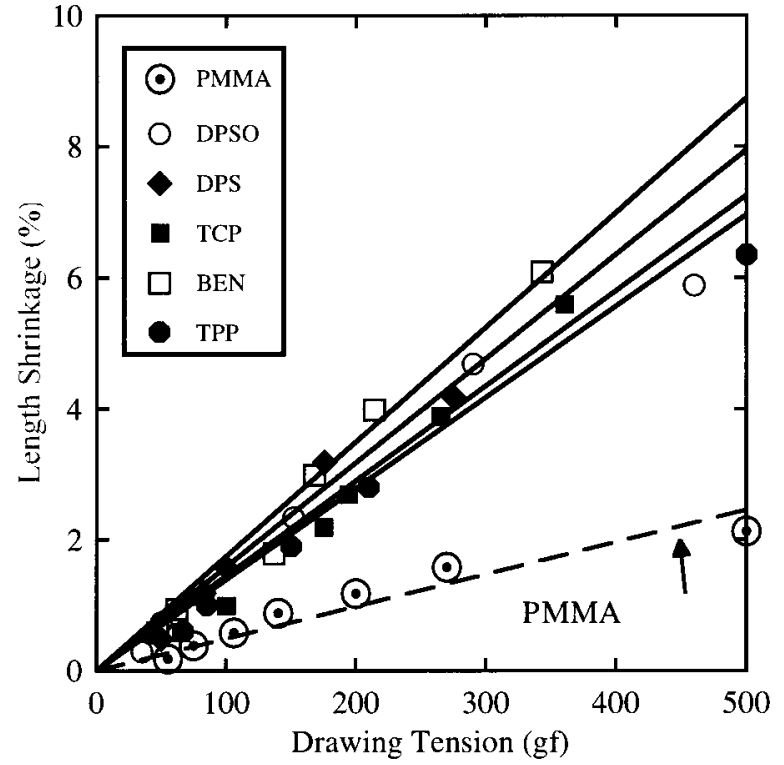

(b)

Figure 5 Relation between the heat-drawing tension and the length shrinkage of the GI POF after $48 \mathrm{~h}$ aging at $70^{\circ} \mathrm{C}$. (a) Dopant concentration in the GI POF is 11 wt \%; (b) Dopant concentration in the GI POF is 20 wt \%. 


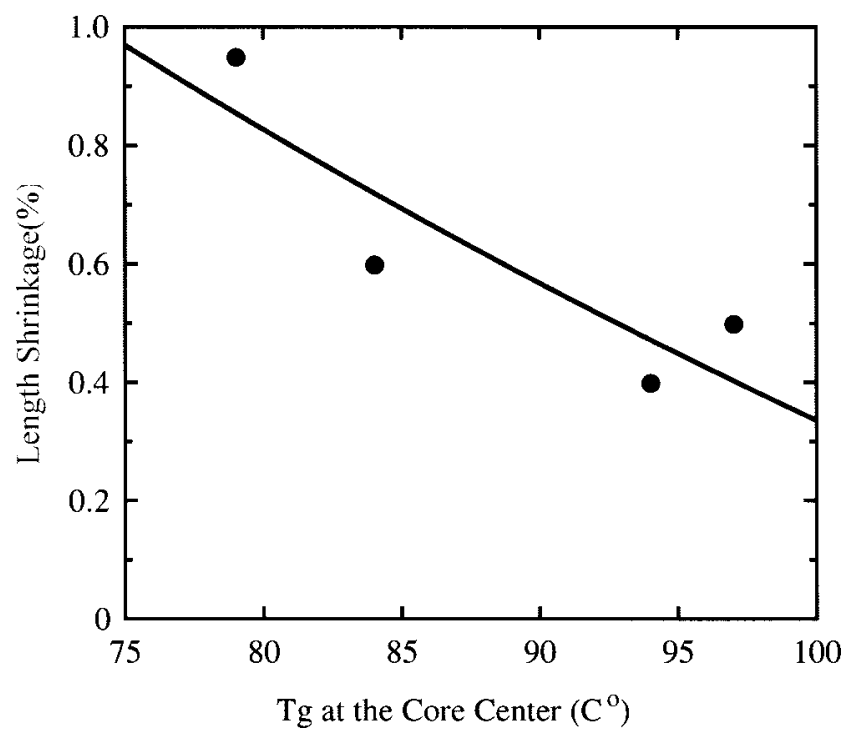

Figure 6 Relation between the $T_{g}$ at the core center and the length shrinkage of the GI POF after $48 \mathrm{~h}$ aging at $70^{\circ} \mathrm{C}$. The heat-drawing tensions of all the GI POFs are almost the same (200 gf).

the GI POF with a low drawing tension. To satisfy both requirements - a tensile strength higher than $1000 \mathrm{kgf}$ (JIS standard) and length shrinkage smaller than $1 \%$ the GI POF doped with 11 wt \% of dopant should be drawn at a tension lower than $100 \mathrm{gf}$.

\section{Optimum preparation conditions of the PMMA- based GI POF}

Figure 7 and 8 show the relations between the fiberdrawing tension and the yield strength and the elongation at break, respectively. The dopant concentrations of the GI POFs are $11 \mathrm{wt} \%$. The yield strength

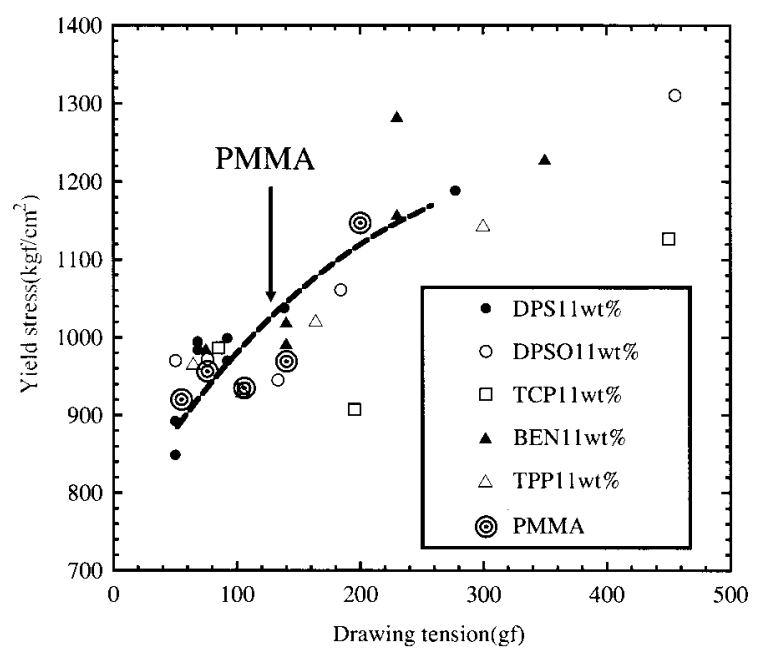

Figure 7 Relation between the heat-drawing tension and the yield strength of the GI POF. Dopant concentration in the GI POF is $11 \mathrm{wt} \%$.

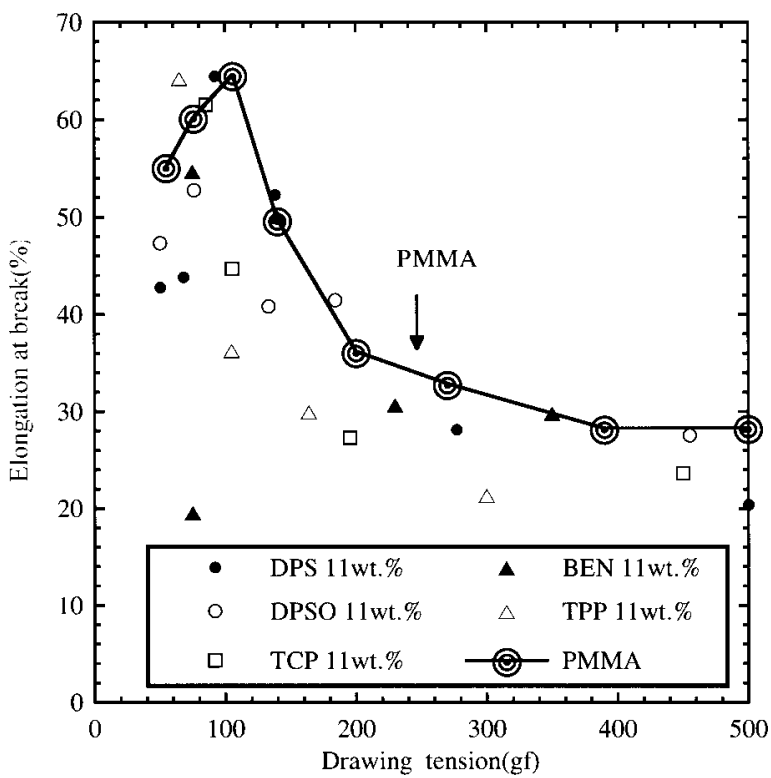

Figure 8 Relation between the heat-drawing tension and the elongation at break of the GI POF. Dopant concentration in the GI POF is $11 \mathrm{wt} \%$.

increases with increased drawing tension of the GI POF, which is almost the same as the relation shown in Figure 3, whereas the elongation at yield is almost independent of the drawing tension, which is also observed in the stress-strain curves shown in Figure 3.

A remarkable relation is observed in Figure 8. The elongation at break shows the maximum peak at approximately 100 gf of drawing tension of the PMMA homopolymer and at a slightly lower tension (80 gf) in the GI POF doped with $11 \mathrm{wt} \%$ dopant. If the GI POF is drawn at a tension lower than $80 \mathrm{gf}$, the polymer chains in the GI POF are not oriented in the axial direction; consequently the GI POF becomes brittle. On the other hand, the polymer chains in the GI POF drawn at a tension higher than $80 \mathrm{gf}$ are fully oriented, and are thus not further elongated.

The detailed experimental analyses mentioned above lead to the optimum preparation conditions for the PMMA-based GI POF to have sufficiently high mechanical strength. The most important factor influencing the mechanical strength of the GI POF was found to be the drawing tension. Approximately 80-gf tension makes the GI POF not only very flexible but also very tensile. In Figure 9 and 10, the stress-strain curve and the length shrinkage during aging are shown, respectively, for the case of the $11 \mathrm{wt} \%$ DPS doped GI POF drawn at nearly optimum tension. At this level of doping, no serious length shrinkage is observed during aging, as shown in Figure 10. In Figure 10, the length shrinkage of the commercially available SI POF is also shown as a comparison. It is noteworthy that the length shrinkage of the SI POF during aging is larger than the GI POF drawn at the optimum tension. Furthermore, the tensile strength ex- 


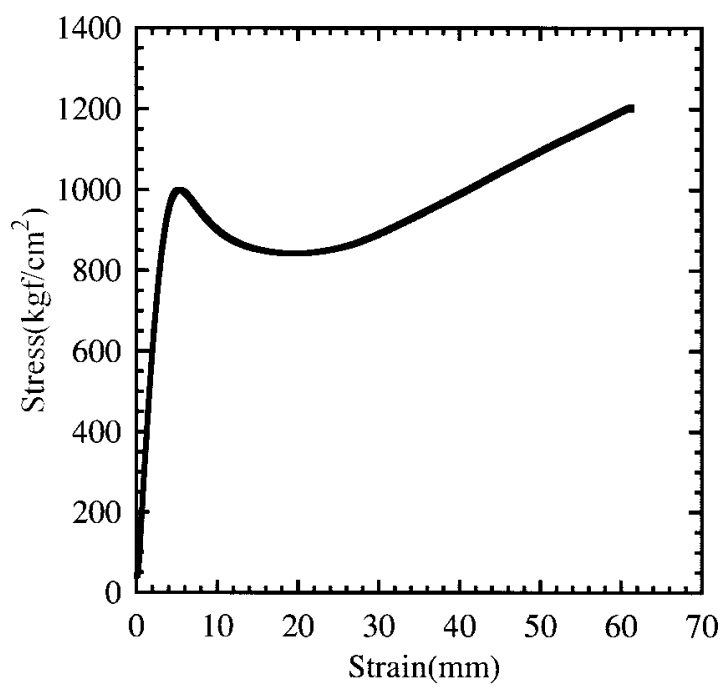

Figure 9 Stress-strain curve of the GI POF drawn at the optimum tension.

ceeds $1000 \mathrm{~kg} / \mathrm{cm}^{2}$, which is higher than the JIS-specified value for the commercially available SI POF. The elongation at break of the GI POF was $50 \%$, which is almost the same as that of the maximum value in the PMMA homopolymer fiber (no dopant) as shown in Figure 8. Compared to the elongation at break of the SI POF shown in Figure 3, that of the GI POF is slightly lower. We found that this difference in the elongation at break between the SI POF and GI POF emerged from the difference in the mechanical properties of cladding. This issue will be described in detail elsewhere. ${ }^{7}$

Figure 11 shows a photograph of the GI POF that was bent with a less than 10-mm bending radius. Because of the excellent mechanical flexibility, such a tight bending

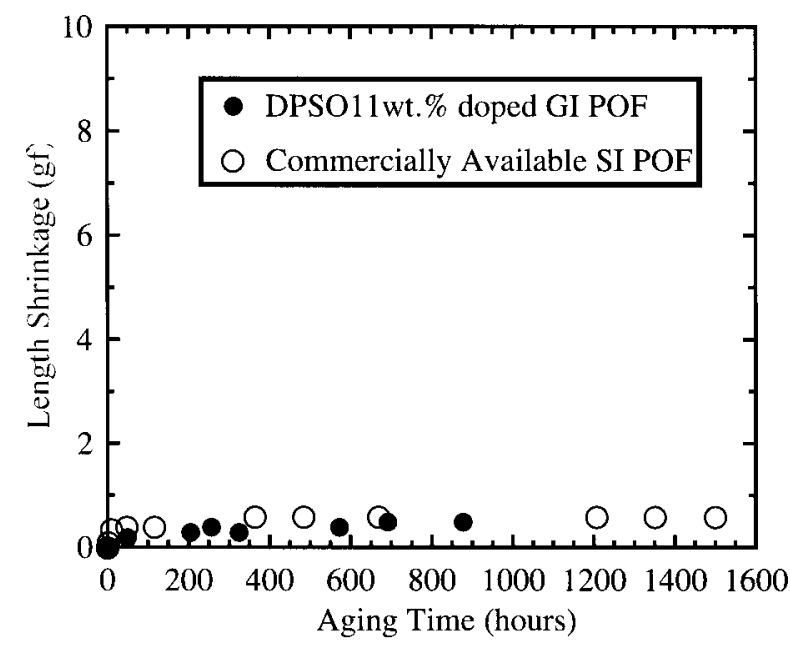

Figure 10 Comparison of the length shrinkage of both GI and SI POFs during aging at $70^{\circ} \mathrm{C}$. The GI POF was drawn at the optimum drawing tension.

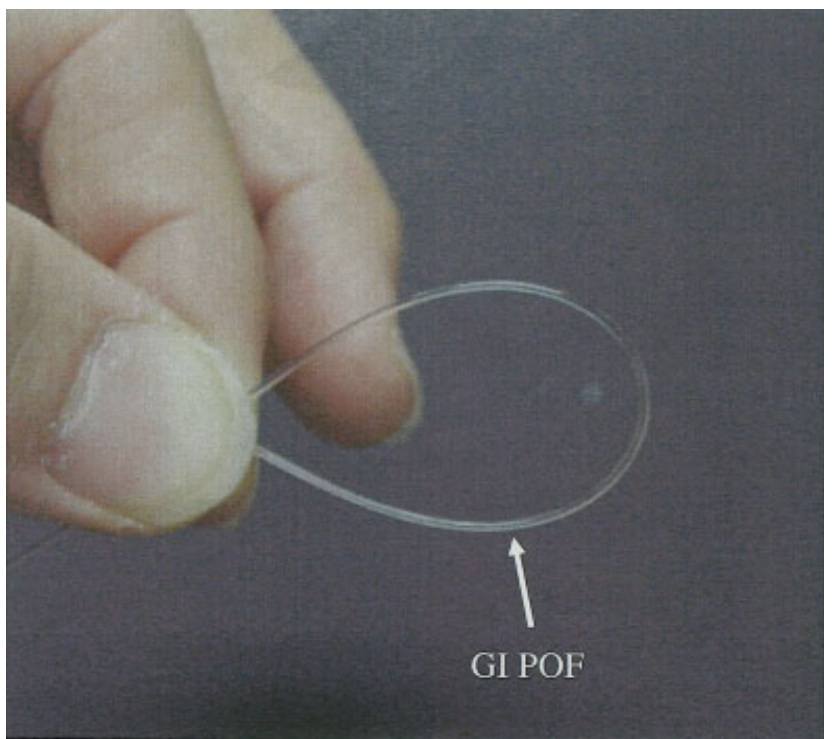

Figure 11 Photograph of the GI POF statically bent with less than $10-\mathrm{mm}$ bending radius.

condition is acceptable in the GI POF link, which is difficult with conventional silica optical fibers. It was already found that a high numerical aperture GI POF has sufficiently low bending loss even if the bending radius is $10 \mathrm{~mm}$. ${ }^{8}$ These remarkable characteristics of the GI POF enable the much easier installation of high-speed optical networks even in the home and office compared with silica-based optical fiber.

\section{CONCLUSIONS}

We have shown that doping in a GI POF has little effect on its mechanical strength parameters (tensile strength, yield strength, elongation at break, and elongation at yield). If a GI POF is drawn from the preform at the optimum tension $(\sim 80 \mathrm{gf})$, a GI POF with high tensile strength $\left(>1000 \mathrm{kgf} / \mathrm{cm}^{2}\right)$, large elongation at break $(>50 \%)$, and little length shrinkage $(<1 \%)$ after aging at $70^{\circ} \mathrm{C}$ was successfully obtained. The GI POF also had sufficiently good flexibility that it could be statistically bent with a 10-mm bending diameter. This high flexibility of the GI POF, while maintaining a large-core diameter, will open the way for the improved ease of installation of a GI POF network even in the home and office.

\section{References}

1. Epworth, R. E. In: Proceedings of the 4th European Conference on Optical Comm., Genoa, Italy, September 1978; p 492.

2. Ishigure, T.; Nihei, E.; Koike, Y. Appl Opt 1994, 33, 4261.

3. Koike, Y.; Ishigure, T.; Nihei, E. J Lightwave Technol 1995, 37, 1475.

4. Ishigure, T.; Sato, M.; Takanashi, O.; Nihei, E.; Nyu, T.; Yamazaki, S.; Koike, Y. J Lightwave Technol 1997, 15, 2095.

5. Kitazawa, M. POF Data Book; MRC Technology: Tokyo, 1993.

6. Sato, M.; Ishigure, T.; Koike, Y. J Lightwave Technol 2000, 8, 952.

7. Ishigure, T.; Sato, M.; Koike, Y. Unpublished results.

8. Ishigure, T.; Sato, M.; Hirai, M.; Koike, Y. J Appl Polym Sci, to appear. 